\title{
Safe, flexible and efficient sheet metal forming: formability - fracture, incremental sheet forming \& rolling
}

\author{
Dorel Banabic $^{1}$ • Anne-Marie Habraken ${ }^{1}$ • Jeong Whan Yoon ${ }^{1}$
}

Published online: 24 June 2015

(C) Springer-Verlag France 2015

The 9th International Conference and Workshop on Numerical Simulation of 3D Sheet Metal Forming Processes (NUMISHEET) was held at the Hilton on the Park in Melbourne, Australia on Jan. 5-10, 2014. This conference series has been established as an international forum to assess the validity and the progress of state-of-the-art simulations as compared to carefully conducted experiments and, thus, to provide vision and strategy for further development of the sheet metal forming process modelling

The present conference was organized by Deakin and Swinburne Universities together. It consisted of over 250 presentations conducted in five parallel sessions over a period of four days. NUMISHEET 2014 was attended by approximately 300 participants. The conference included scientists, engineers and software developers from academia, research institutions and industry worldwide, and provided a unique forum for stimulating discussions and exchanges of new ideas pertaining to numerical methods, material behaviour, process modelling and forming technologies.

A unique feature of NUMISHEET that distinguishes it from other metal forming conferences is the NUMISHEET Benchmark Study, in which participants are challenged prior to the conference to predict the results of specified manufacturing processes. Four benchmarks were organized: BM-1: Nonlinear Strain Path Forming Limit of a Reverse draw, BM-2: Springback of draw-redraw pan, BM-3: Incremental sheet forming, BM-4: Wrinkling during redraw.

Dorel Banabic

banabic@tcm.utcluj.ro

1 Technical University of Cluj Napoca, Cluj Napoca, Romania
The Auto Steel Partnership of North America including Chrysler, GM, Ford, USA Steel Corporation, ArcelorMittal (BM-1,2), Boeing (BM-3), and Alcoa (BM-4) were served as the benchmark chairs and co-chairs closely collaborating with academics. This was the first conference to involve the aerospace industry in a benchmark study

The concept of virtual manufacturing has been developed in order to increase the industrial performances, being one of the most efficient ways of reducing the manufacturing times and improving the quality of the products. Numerical simulation of metal forming processes, as a component of the virtual manufacturing process, has a very important contribution to the reduction of the lead time. The finite element method is currently the most widely used numerical procedure for simulating sheet metal forming processes. The accuracy of the simulation programs used in industry is influenced by the constitutive models and the forming limit models incorporated in their structure. In its first part, this thematic issue is concerned with the formability of metallic materials which generate both academic and practical interest. Eight articles dedicated to formability and fracture were selected from the conference which bring to fore current scientific aspects in the fields such as analysis, modelling and experimental determination of formability of metallic materials.

The second part of this thematic issue is focused on flexible sheet forming technology including roll forming and incremental sheet forming. About roll forming, an in line shape compensation method for high strength steel is presented as well as a micro rolling research for ultra-thin strip. For incremental forming, different topics are investigated such as process speed, accuracy, optimization, formability issues and both predictive character and CPU time efficiency of FE simulations. 
Incremental sheet forming is an effective metal forming technique without using die for small and medium batch manufacturing. Due to the various advantages including the reduced production cost and time of prototypes, improved formability, easy modification of part design etc., this flexible forming process is being popular as a new innovative forming technology. This second part includes nine articles selected from the conference. Current scientific aspects such as scale effect, control, process accuracy and numerical models of Incremental Sheet Forming \& Roll Forming are studied. All the articles of this thematic issue were peer-reviewed in accordance with the editorial policies of the Journal.

June 4, 2015

Acknowledgments The success of this conference, as well as this special issue, primarily depends on all the participants to whom we express our sincere thanks. We also acknowledge the contributions of the conference organizing committee members, together with the financial support of the NUMISHEET 2014 sponsors. Finally, we acknowledge Prof. Francisco Chinesta (Editor-in-Chief for Int. J. Mater Forming), who is the driving force behind the success of this Special Issue. 